\title{
Hydric-Agent: Ferramenta de Simulação Baseada em Agentes para Gestão da Água em Áreas Residenciais
}

\author{
Fernando L. Alencar ${ }^{1}$, Diana J. Monsalve-Herrera ${ }^{2}$, Carolina G. Abreu ${ }^{1}$, \\ Cassio G. C. Coelho ${ }^{1}$, Conceição de Maria A. Alves ${ }^{2}$, Célia G. Ralha ${ }^{1}$ \\ ${ }^{1}$ Programa de Pós-graduação em Informática, Departamento de Ciência \\ da Computação, Universidade de Brasília (UnB), DF, Brasil \\ ${ }^{2}$ Programa de Pós-graduação em Tecnologia Ambiental e Recursos Hídricos, \\ Departamento de Engenharia Civil e Ambiental, UnB , DF, Brasil \\ \{carolabreu, calves, ghedini\}@unb.br
}

\begin{abstract}
In the implementation of water management plans, it is necessary to understand the behavior of water consuming agents in relation to decisions, plans and projects of water system management. This integration allows decision making by managers in accordance with the reality of the water system. In the present work we present the Hydric-Agent tool developed in JADEX that allows the simulation of water consumers under different water management scenarios, providing the user of the platform with a view of the cooperativism of the water consumer agent and the degree of adhesion to the action of management.
\end{abstract}

Resumo. Na implementação de planos de gerenciamento hídrico, se faz necessário compreender o comportamento dos agentes consumidores de água diante de decisões, planos e projetos de gerenciamento do sistema hídrico. Essa integração possibilita tomadas de decisão por parte dos gestores em concordância com a realidade do sistema hídrico. No presente trabalho se apresenta a ferramenta Hydric-Agent desenvolvida em JADEX que permite a simulação de consumidores de água sob diferentes cenários de gestão hídrica, fornecendo ao usuário da plataforma uma visão do cooperativismo do agente consumidor de água e o grau de adesão à ação de gestão estabelecida.

\section{Introdução}

A gestão de sistemas hídricos urbanos se desenvolve com ênfase em processos hidrológicos, econômicos, políticos e sociais. A complexidade das leis que governam esses processos e as ações que resultam das interações entre eles proporcionam propriedades aos sistemas hídricos urbanos que permitem sua caracterização como sistemas complexos adaptativos [Mitchell 2009, Sichman 2015]. A operação de um sistema hídrico urbano envolve a participação de atores como consumidores de água, companhias de saneamento básico e agências reguladoras desse serviço público. A dinâmica e a possibilidade de aprendizado, de evolução e de adaptação de atores envolvidos no funcionamento desses sistemas resultam em comportamentos não determinísticos que caracterizam sistemas complexos. Considerar o comportamento desses atores na simulação de sistemas hídricos urbanos pode contribuir para definição de programas (medidas e ações) de gestão mais efetivos e adaptados à realidade local [Giacomoni et al. 2013, Kanta and Zechman 2013]. 
Os sistemas hídricos abordados como sistemas complexos adaptativos(SCA) podem ser simulados por meio de modelos baseados em agentes (MBA) [Holland 1995], uma vez que esses são considerados como uma metáfora natural para a representação de sistemas complexos. Os MBAs, por sua vez, podem ser representados por meio de Sistemas Multiagentes (SMA), área de Inteligência Artificial Distribuída. SMA permite a implementação de simulações com ênfase nas ações e interações de agentes em um ambiente computacional.

Um dos usos de SMA é representar o raciocínio e o conhecimento de agentes heterogêneos que interagem uns com os outros, cooperativamente, em busca de um objetivo global. O Hydric Agent é uma ferramenta de SMA, baseada na simulação de modelos baseados em agentes (MBA), que visa a auxiliar o gestor do setor de recursos hídricos a selecionar ações e medidas de gestão de sistema hídrico de acordo com o perfil comportamental da comunidade ou de consumidores de água. Por meio da aplicação do Hydric Agent é possível integrar ou inserir o comportamento do usuário em modelos de sistemas hídricos que geralmente representam apenas processos hidráulicos e hidrológicos sem levar em consideração a influência do componente social no comportamento desses sistemas. O objetivo deste trabalho é apresentar a ferramenta Hydric Agent e seu processo de construção e implementação. Complementarmente, serão ilustradas algumas possibilidades de análises que resultaram de sua aplicação a um estudo de caso na comunidade de Brazlândia, Distrito Federal.

\section{Hydric-Agent}

Nessa sessão serão apresentados alguns fundamentos e métodos que foram utilizados na construção da proposta de ferramenta do Hydric-Agent. Uma detalhada pesquisa de campo foi realizada para que os agentes representassem de forma mais realista o comportamento de cidadãos da região de estudo. Por fim, os detalhes da modelagem conceitual e arquitetural do Hydric-Agent são apresentados.

\subsection{Conceitos}

Sob o ponto de vista da computação, um agente é uma entidade autônoma inteligente dotada de sensores e de atuadores, que a permitem acessar o ambiente em que se encontra e atuar sobre ele. Outras características inerentes aos agentes, como proatividade, mobilidade e comunicação proporcionam a eles a capacidade de adaptação a mudanças ambientais, considerando não apenas as percepções do meio em que se encontram, mas também objetivos próprios que eles desejam alcançar [Russel and Norvig 2010]. O Projeto de construção de um agente pode ser descrito como PAGE (Perceptions, Actions, Goals e Environment). O agente percebe e interage com o ambiente. O nível de complexidade de um SMA é dado principalmente pela variedade e completude do ambiente.

A cognição dos agentes pode ser projetada de diferentes meios. Uma das opções é o modelo mentalista baseado em Crença-Desejo-Intenção ou Belief-Desire-Intention (BDI). O modelo é composto por uma arquitetura deliberativa no qual o estado interno de um agente pode ser descrito por conjuntos de estados mentais [Bratman 1987]. As crenças representam aquilo que o agente pode perceber e registrar do ambiente além de percepções internas a seu próprio respeito. Os desejos são o conjunto de atitudes mentais que motivam o planejamento do agente a realizar suas metas. As intenções definem os planos que serão executados para a realização de um objetivo [Wooldridge 2009]. 
Os planos são conjuntos ordenados de ações resultantes de um processo de raciocínio prático constituído de duas etapas: deliberação e planejamento (ou raciocínio meios-fim). Na etapa de deliberação, o agente acessa os estados atuais de suas crenças e de outras variáveis internas, como mensagens, para decidir qual o objetivo deve ser admitido como corrente no momento. Na etapa subsequente, planejamento, o agente toma o resultado da etapa anterior para analisar que ações são possíveis de serem tomadas em uma ordem lógica para alcançar o objetivo atual [Wooldridge 2009].

\subsection{Levantamento de Dados}

Para viabilizar a definição do comportamento dos agentes no simulador Hydric-Agent, uma pesquisa de campo foi conduzida. O levantamento das crenças e atitudes de cidadãos reais foi realizada a partir de 320 questionários aplicados na região de interesse [Monsalve-Herrera 2018]. Esse material permitiu a definição do modelo conceitual dos agentes, com base no framework $i *$ [Yu 1995]. A caracterização das percepções, ações, objetivos e o ambiente foram extraídas dos questionários, com o auxílio de especialistas, resultando na configuração do comportamento dos agentes BDI-cognitivos.

Os agentes foram considerados como domicílios consumidores de água, que podem comportar-se de forma cooperativa ou não cooperativa. Os domicílios foram categorizados em três classes de rendas: renda alta (mais que 10 salários mínimos); renda média (2 a 4 salários mínimos); e renda baixa (menos que 2 salários mínimos). Cada domicílio também foi caracterizado conforme a escolaridade: nível fundamental, médio e superior completos e incompletos.

Agentes não cooperativos caracterizam-se por não se adaptar às regras de consumo de água estabelecidas pelo gestores e não diminuirem a quantidade de água consumida, podendo ainda, aumentar seu consumo de água. Já os agentes do tipo cooperativo adaptam-se às regras de consumo de água estabelecidas pelo gestor e diminuem a quantidade de água consumida como resposta a medidas e ações de gestão impostas pelo gestor. As possíveis medidas de gestão de demanda de água implantadas são: campanhas educativas e implantação de tarifa de contingência.

\subsection{Arquitetura e Implementação}

A partir do levantamento de dados e definição do modelo conceitual foi construída a ferramenta Hydric-Agent ${ }^{1}$ utilizando como middleware o framework JADEX [Braubach and Pokahr 2012] e a linguagem de programação Java. Foi proposta uma arquitetura em três camadas com hierarquia híbrida, ou seja, as mudanças de comportamento podem ser acionadas diretamente pela alteração de uma medida do gestor (hierarquia top-down) ou pela tomada de decisão individual de cada agente consumidor (bottom-up). Os requisitos do funcionamento da ferramenta Hydric-Agent também são derivados do modelo conceitual e a implementação baseou-se na modelagem do SMA por meio da metodologia Tropos [Bresciani et al. 2004]. A visão geral pode ser observada na Figura 1. Percebe-se a definição de um agente gestor e de agentes consumidores, de diferentes tipologias. Cada tipologia determina como os agentes consumidores irão interagir com o recurso água conforme as suas crenças (renda, taxa de escolaridade, etc). A implementação dos agentes segue o conceito PAGE, conforme apresentado na Tabela 1.

\footnotetext{
${ }^{1}$ Código-fonte disponível em: https://github.com/MASE-UnB/Hydric-Agents-BDI
} 


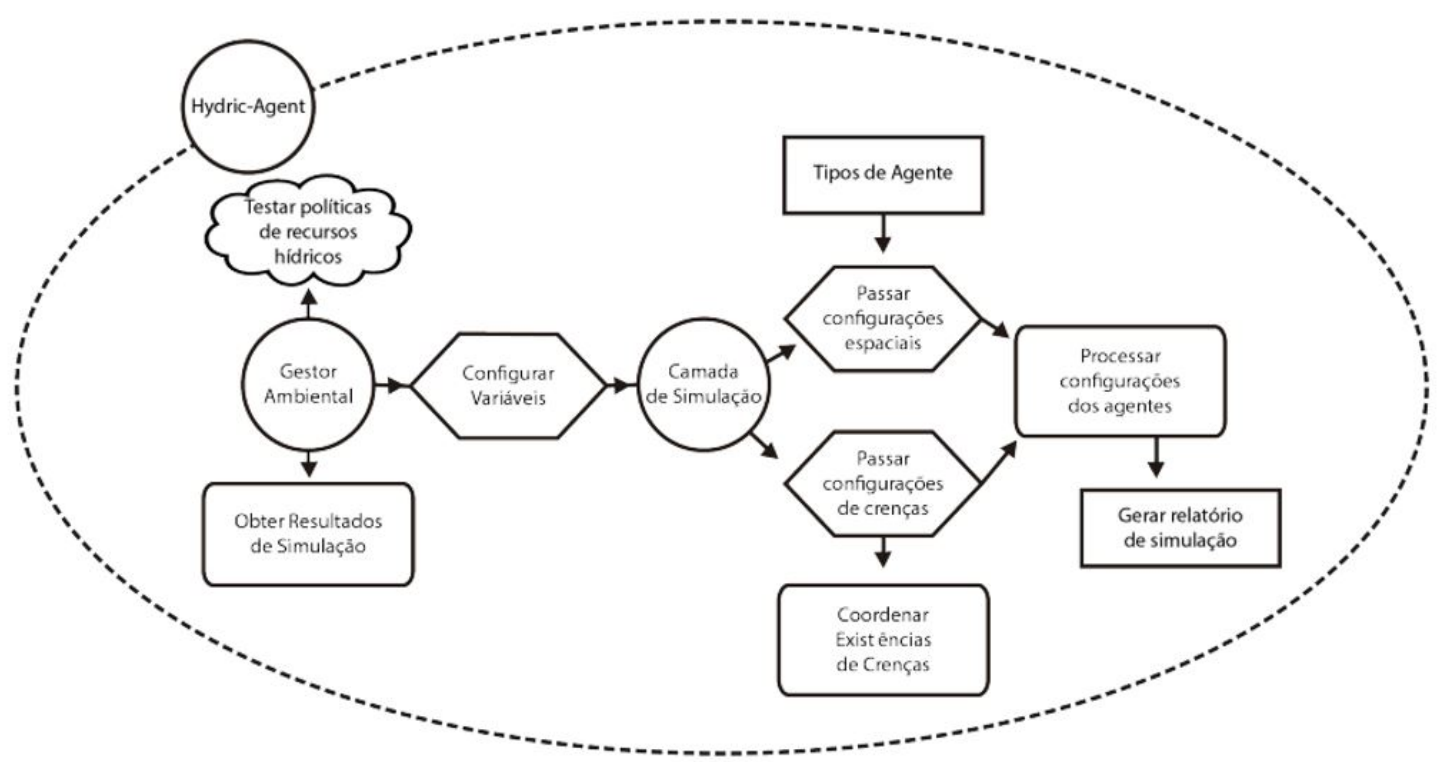

Figura 1. Diagrama Tropos que representa o Projeto Arquitetural Hydric-Agent

Tabela 1. PAGE utilizado para desenvolvimento da plataforma Hydric-Agent

\begin{tabular}{l|l}
\hline Percepções & $\begin{array}{l}\text { Campanhas educativas } \\
\text { Tarifa } \\
\text { Estação de chuva ou seca }\end{array}$ \\
\hline Ações & $\begin{array}{l}\text { Economia de Água } \\
\text { Desperdício de Água }\end{array}$ \\
\hline Objetivos & $\begin{array}{l}\text { Satisfazer as necessidades } \\
\text { mensais de uso de água }\end{array}$ \\
\hline Ambiente & $\begin{array}{l}\text { Área residencial urbana } \\
\text { Grid de simulação }\end{array}$ \\
\hline
\end{tabular}

A Tabela 2 apresenta a classificação do ambiente real esperado de uma área residencial urbana em contraste com o ambiente computacional modelado na ferramenta Hydric-Agent. Esse ambiente modelado é o que será percebido pelos agentes computacionais que possuem crenças baseadas no estudo de campo e apresentam mecanismo deliberativo-cognitivo construído conforme o modelo BDI.

No Hydric-Agent foram construídas três camadas: interface, controle e física. A camada de interface fornece ao usuário a visualização do cenário em forma de grid e as opções de configurações do sistema (Figura 2). Por meio dessa camada o usuário pode configurar o tamanho do grid (Configure Grid), adicionar ou excluir agentes (através de cliques nas células do grid), alterar o ambiente com a inserção ou remoção das crenças (Toggle Tax e Toggle Education), controlar os steps da simulação (Next Step), iniciar e pausar a execução do software (Start/Stop) e gerar o relatório da simulação (Generate Report). Na camada de controle, as configurações definidas através da Camada de Interface são utilizadas para execução da simulação. A organização e composição hierárquica dos agentes, assim como os mecanismos de comunicação deles e seus respectivos PAGE são descritas nessa camada. Na camada física atuam os agentes consumidores de água o 
modelo de raciocínio BDI específico para cada agente. Essa camada é a responsável por gerar todos os dados de consumo dos agentes do Sistema multiagente e enviá-los para a camada de controle para que possam ser transcritos em formato de relatório. A ferramenta produz dados de consumo de água dos agentes e lista o número de agentes cooperativos por renda e por escolaridade. Esses dados podem ser utilizados como cenário inicial de ferramentas de suporte à decisão de recursos hídricos, oferecendo uma visão holística do sistema a ser avaliado pelo gestor da água.

Tabela 2. Classificação do Ambiente

\begin{tabular}{c|c}
\hline Real & Modelado \\
\hline \hline Parcialmente observável & Parcialmente observável \\
\hline Estocástico & Determinístico \\
\hline Sequencial & Episódico \\
\hline Dinâmico & Estático \\
\hline Contínuo & Discreto \\
\hline Multiagente & Multiagente \\
\hline Híbrido & Híbrido \\
\hline
\end{tabular}
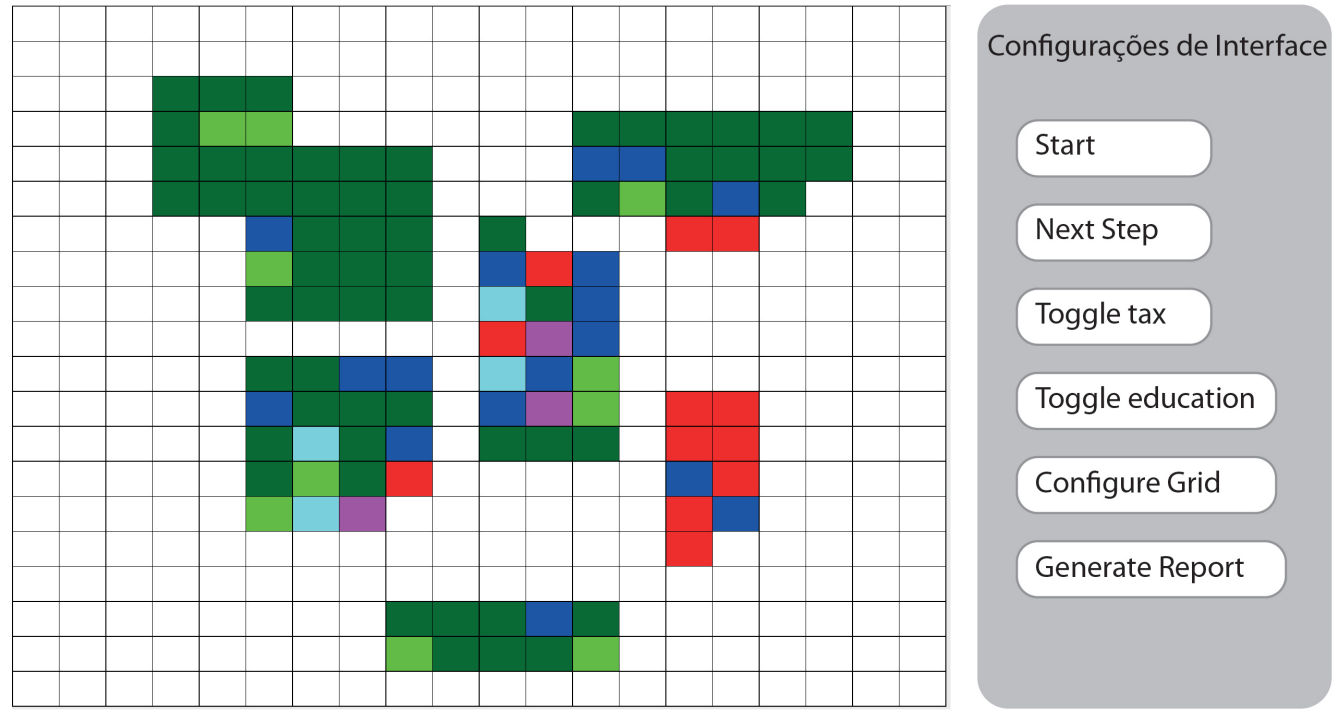

Figura 2. Interface e Grid de Simulação Hydric-Agent

A simulação foi configurada para que cada passo represente um mês de consumo de água, uma vez que há diferença nas políticas aplicadas durante os meses secos e chuvosos. Os agentes são distribuídos pelo grid sendo que suas cores e tonalidade mostram o cooperativismo de cada agente por tipo de renda como apresentado na Figura 3. Nessa versão do Hydric-Agent, considerou-se não haver influência de vizinhança nos padrões de consumo de água residencial urbana, ou seja, residentes não tomam decisão de consumir por influência de vizinhos.

\section{Estudo de Caso: Consumo de Água Residencial Urbano}

A presente situação de crise hídrica no DF resultou na adoção de medidas de racionamento de água para a maioria das regiões administrativas. Os níveis dos principais reservatórios de abastecimento da região, Descoberto e Santa Maria, atingiram em 2017 os 
menores índices do registro histórico. Como estudo de caso foi escolhida a área urbana da Região Administrativa IV do DF, Brazlândia. A represa do Rio Descoberto e a formação do Lago Descoberto, que antes faziam parte de fazendas da região de Brazlândia, hoje são responsáveis pelo abastecimento de mais de $60 \%$ da água de todo o DF. A Figura 4 apresenta a visão geral da área urbana, utilizada como guia para construção do grid de simulação Hydric-Agent.

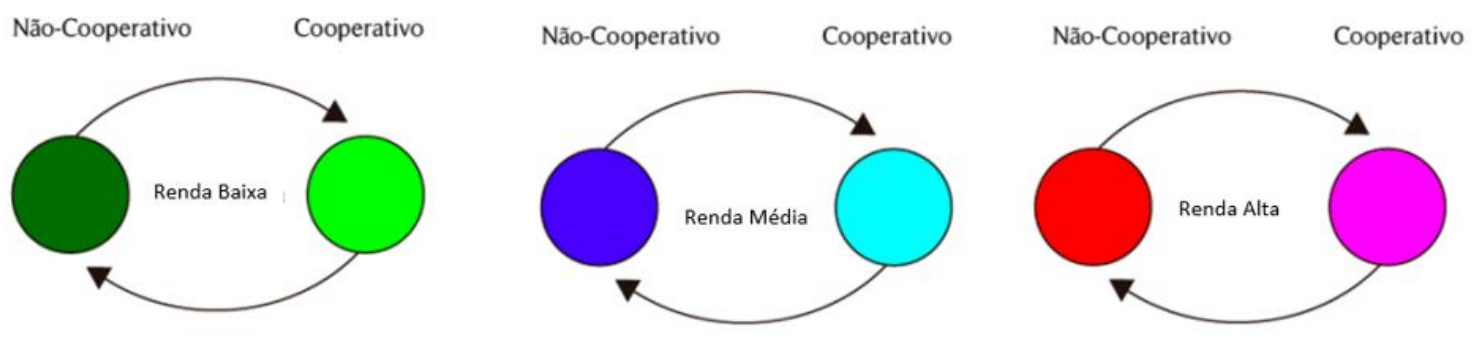

Figura 3. Representação de categoria e comportamento dos agentes

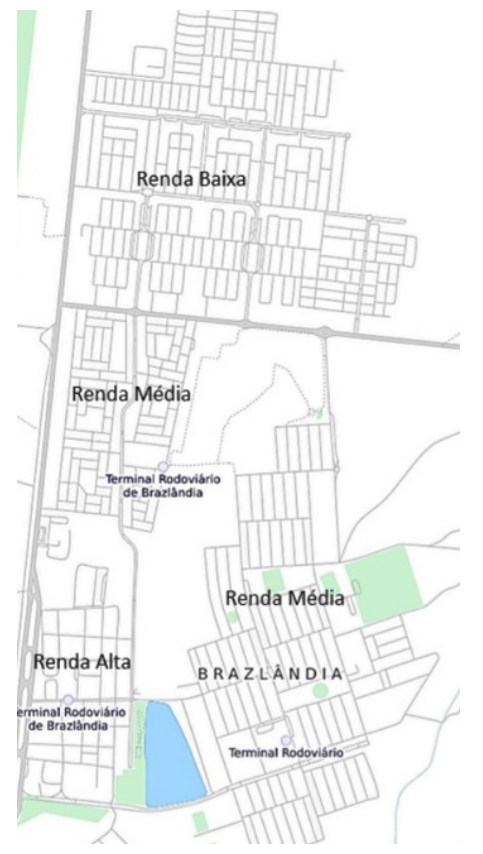

Figura 4. Área residencial urbana da Região Administrativa de Brazlândia, DF

A simulação do Hydric-Agent em Brazlândia permite avaliar a adesão dos consumidores da região às ações da gestão por meio da representação do comportamento cooperativo ou não cooperativo. A área urbana e a mudança de comportamento dos agentes pode ser visualizada quando o usuário acompanha as células do grid, que correspondem às residências, e as cores, que correspondem o tipo de renda e o perfil de cooperação (Figura 3).

\subsection{Experimentos e Resultados}

As simulações e relatórios produzidos pelo Hydric-Agent permitem observar o comportamento cooperativo de agentes classificados por renda e por escolaridade mensalmente, bem como os respectivos consumos de água. Um exemplo de relatório do Hydric-Agent 
está apresentado na Figura 5 que ilustra a porcentagem de agentes de renda baixa (de maior representatividade em Brazlândia), por escolaridade, que apresentaram ação cooperativa após campanhas educativas ao longo do ano (linhas coloridas). Os resultados podem ser comparados com a porcentagem de agentes de renda baixa, por escolaridade, que apresentaram ação cooperativa quando não havia campanhas educativas (sem gestãoSG) (barras coloridas).

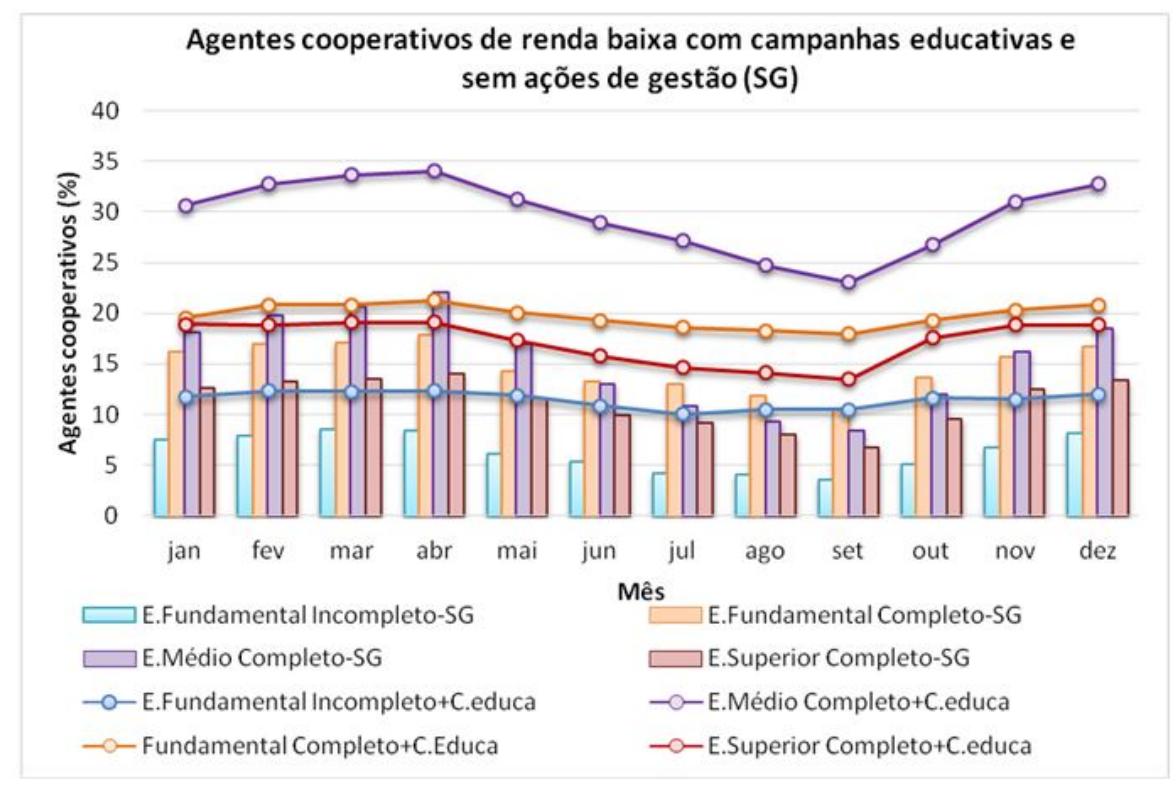

Figura 5. Porcentagem de agentes cooperativos de renda baixa sem ações de gestão (SG) e implementando campanhas educativas simulado em Hydric-Agent

A Figura 5 mostra que consumidores de renda baixa são receptivos a campanhas educativas pois as porcentagens de agentes cooperativos são maiores para todas escolaridades ao longo do ano, quando comparadas com porcentagens da simulação sem ação de gestão (SG). A observação da variação do cooperativismo ao longo do ano permite também avaliar a percepção do consumidor em relação à disponibilidade de água (representada pela ocorrência do período de chuva, outubro a abril). No período de estiagem, maio a setembro, em média há redução de cooperativismo em todas as categorias de renda, mas para a renda baixa essa redução não é acentuada devido a característica de domicílios dessa categoria, por exemplo, a inexistência de grandes áreas de jardins que requeiram irrigação. A funcionalidade do Hydric-Agent também auxilia no entendimento da influência da escolaridade na efetividade de diferentes ações de gestão de água. Análises similares podem ser feitas para as demais categorias de renda, que não foram aqui apresentadas por restrição de espaço. A ferramenta Hydric Agent mostrou-se útil para apoio à seleção de medidas e ações de gestão da água permitindo adaptar as políticas à realidade local.

\section{Conclusão}

Com a finalidade de propor uma ferramenta que permita auxiliar o gestor recursos hídricos na tomada de decisões relacionadas à gestão de recursos hídricos, o Hydric-Agent propicia uma melhor visualização dos cenários de uso de água urbano. A ferramenta considera 
o comportamento do usuário de recursos hídricos para avaliar diferentes estratégias de gestão hídrica adaptativa. As tecnologias utilizadas para implementação foram adequadas por prover bibliotecas e interfaces que facilitam o processo de comunicação entres as entidades do programa, possibilitando uma representação explícita do ambiente e dos objetivos que os agentes podem alcançar. Os relatórios gerados a partir das simulações dos agentes, permitiram a plotagem de gráficos e a análise do comportamento do usuários de recursos hídricos do cenário de estudo. A aplicação Hydric-Agent foi capaz de provarse dinâmica e útil para a utilização por gestores ambientais ou pesquisadores de áreas interdisciplinares. É ferramenta de apoio à tomada de decisão capaz de promover o entendimento da dinâmica do sistema hídrico e de seus agentes, ao criar um ambiente computacional capaz de simular as necessidades ambientais, econômicas e sociais.

\section{Referências}

Bratman, M. E. (1987). Intention, Plans, and Practical Reason. Harvard University Press, Cambidge, MA.

Braubach, L. and Pokahr, A. (2012). Jadex active components framework-bdi agents for disaster rescue coordination. Software agents, agent systems and their applications, $32: 57-84$.

Bresciani, P., Perini, A., Giorgini, P., Giunchiglia, F., and Mylopoulos, J. (2004). Tropos: An Agent-Oriented Software Development Methodology. Autonomous Agents and MultiAgent Systems, 8(3):203-236.

Giacomoni, M., Kanta, L., and Zechman, E. (2013). Complex Adaptive Systems Apporach to Simulate the Sustainability of Water Resources and Urbanization. Journal of Water Resources Planning and Management, 139(June):554-564.

Holland, J. H. (1995). Hidden Order: How Adaptation Builds Complexity. Addison Wesley Longman Publishing Co., Inc., Redwood City, CA, USA.

Kanta, L. and Zechman, E. (2013). Complex Adaptive Systems Framework to Assess Supply-Side and Demand-Side Management for Urban Water Resources. Journal of Water Resources Planning and Management, 140(January):75-85.

Mitchell, M. (2009). Complexity: A Guided Tour. Oxford University Press, USA.

Monsalve-Herrera, D. J. (2018). Modelo comportamental com base em agentes para gestão adaptativa de água: caso de estudo de consumo de água residencial urbana em Brazlândia/DF. PhD thesis, Departamento de Eng. Civil e Ambiental, UnB.

Russel, S. J. and Norvig, P. (2010). Artificial Intelligence: a modern approach. Prentice Hall, 2nd edition.

Sichman, J. S. (2015). Operacionalizacao de sistemas complexos. In Furtado, B. A., Sakowski, P., and Tovolli, M., editors, Modelagem de sistemas complexos para políticas públicas, page 436. Instituto de Pesquisa Economica Aplicada - IPEA.

Wooldridge, M. (2009). Introduction to Multiagent Systems. John Wiley \& Sons Ltd, West Sussex, UK, 2nd edition.

Yu, E. S.-K. (1995). Modelling Strategic Relationships for Process Reengineering. PhD thesis, Department of Computer Science, University of Toronto. 